\title{
Exponential Separations in the Energy Complexity of Leader Election*
}

\author{
Yi-Jun Chang \\ University of Michigan \\ Ann Arbor, MI
}

\author{
Tsvi Kopelowitz \\ University of Michigan \\ Ann Arbor, MI
}

\author{
Seth Pettie \\ University of Michigan \\ Ann Arbor, MI
}

\author{
Ruosong Wang \\ Tsinghua University \\ Beijing, China
}

\author{
Wei Zhan \\ Tsinghua University \\ Beijing, China
}

\begin{abstract}
Energy is often the most constrained resource for battery-powered wireless devices and the lion's share of energy is often spent on transceiver usage (sending/receiving packets), not on computation. In this paper we study the energy complexity of Leader Election and Approximate Counting in several models of wireless radio networks. It turns out that energy complexity is very sensitive to whether the devices can generate random bits and their ability to detect collisions. We consider four collision-detection models: Strong-CD (in which transmitters and listeners detect collisions), Sender-CD and Receiver-CD (in which only transmitters or only listeners detect collisions), and No-CD (in which no one detects collisions.)

The take-away message of our results is quite surprising. For randomized Leader Election algorithms, there is an exponential gap between the energy complexity of Sender-CD and Receiver-CD:
\end{abstract}

$$
\text { No-CD }=\text { Sender-CD } \gg \text { Receiver-CD = Strong-CD }
$$

and for deterministic Leader Election algorithms, there is another exponential gap in energy complexity, but in the reverse direction:

$$
\text { No-CD = Receiver-CD } \gg \text { Sender-CD = Strong-CD }
$$

In particular, the randomized energy complexity of Leader Election is $\Theta\left(\log ^{*} n\right)$ in Sender-CD but $\Theta\left(\log \left(\log ^{*} n\right)\right)$ in Receiver-CD, where $n$ is the (unknown) number of devices. Its deterministic complexity is $\Theta(\log N)$ in Receiver-CD but $\Theta(\log \log N)$ in Sender-CD, where $N$ is the (known) size of the devices' ID space.

There is a tradeoff between time and energy. We give a new upper bound on the time-energy tradeoff curve for randomized Leader Election and Approximate Counting. A critical component

\footnotetext{
*Supported by NSF grants CNS-1318294, CCF-1514383, and CCF-1637546. Research performed while Ruosong Wang and Wei Zhan were visiting University of Michigan. Ruosong Wang and Wei Zhan are supported in part by the National Basic Research Program of China Grant 2015CB358700, 2011CBA00300, 2011CBA00301, the National Natural Science Foundation of China Grant 61202009, 61033001, 61361136003. E-mails: cyijun@umich.edu, kopelot@gmail.com, pettie@umich.edu, wrs13@mails.tsinghua.edu.cn, jollwish@gmail.com
}

Permission to make digital or hard copies of all or part of this work for personal or classroom use is granted without fee provided that copies are not made or distributed for profit or commercial advantage and that copies bear this notice and the full citation on the first page. Copyrights for components of this work owned by others than ACM must be honored. Abstracting with credit is permitted. To copy otherwise, or republish, to post on servers or to redistribute to lists, requires prior specific permission and/or a fee. Request permissions from permissions@acm.org.

STOC'17, Montreal, Canada

(C) 2017 ACM. 978-1-4503-4528-6/17/06 ..\$15.00

DOI: $10.1145 / 3055399.3055481$ of this algorithm is a new deterministic Leader Election algorithm for dense instances, when $n=\Theta(N)$, with inverse-Ackermann-type $(O(\alpha(N)))$ energy complexity.

\section{CCS CONCEPTS}

- Theory of computation $\rightarrow$ Distributed algorithms;

\section{KEYWORDS}

Approximate counting, collision detection, distributed computing, energy efficiency, leader election

\section{ACM Reference format:}

Yi-Jun Chang, Tsvi Kopelowitz, Seth Pettie, Ruosong Wang, and Wei Zhan. 2017. Exponential Separations in the Energy Complexity of Leader Election. In Proceedings of 49th Annual ACM SIGACT Symposium on the Theory of Computing, Montreal, Canada, fune 2017 (STOC'17), 13 pages.

DOI: $10.1145 / 3055399.3055481$

\section{INTRODUCTION}

In many networks of wireless devices the scarcest resource is energy, and the lion's share of energy is often spent on radio transceiver usage $[4,34,38,40]-$ sending and receiving packets-not on computation per se. In this paper we investigate the energy complexity of fundamental problems in wireless networks: Leader Election, Approximate Counting, and taking a Census.

In all the models we consider time to be partitioned into discrete slots; all devices have access to a single shared channel and can choose, in each time slot, to either transmit a message $m$ from some space $\mathcal{M}$, listen to the channel, or remain idle. Transmitting and listening each cost one unit of energy; we measure the energy usage of an algorithm on $n$ devices by the worst case energy usage of any device. For the sake of simplicity we assume computation is free and the message size is unbounded. If exactly one device transmits, all listeners hear the message $m$, and if zero devices transmit, all listeners hear a special message $\lambda_{S}$ indicating silence. We consider four models that differ in how collisions can be detected.

Strong-CD. Each sender and listener receives one of three signals: $\lambda_{S}$ (silence, if zero devices transmit), $\lambda_{N}$ (noise, if $\geq 2$ devices transmit), or a message $m \in \mathcal{M}$ (if one device transmits).

Sender-CD. (Often called "No-CD" [22]) Each sender and listener receives one of two signals: $\lambda_{S}$ (zero or $\geq 2$ devices transmit), or a message $m \in \mathcal{M}$ (if one device transmits). Observe that the Sender-CD model has no explicit collision 
detection, but still allows for sneaky collision detection: if a sender hears $\lambda_{S}$, it can infer that there was at least one other sender.

Receiver-CD. Senders receive no signal. Each listener receives one of three signals: $\lambda_{S}$ (silence, if zero devices transmit), $\lambda_{N}$ (noise, if $\geq 2$ devices transmit), or a message $m \in \mathcal{M}$ (if one device transmits).

No-CD. Senders receive no signal. Listeners receive one of two signals: $\lambda_{S}$ (zero or $\geq 2$ devices transmit) or a message $m \in \mathcal{M}$. To avoid trivial impossibilities, it is promised that there are at least 2 devices.

In addition we distinguish randomized and deterministic algorithms. In the randomized model all $n$ devices begin in exactly the same state ( $n$ is unknown and unbounded), and can break symmetry by generating private random bits. In the deterministic model we assume that all $n$ devices have unique IDs in the range [N] $\stackrel{\text { def }}{=}\{0, \ldots, N-1\}$, where $N$ is common knowledge but $n \leq N$ is unknown.

We consider the following fundamental problems.

Leader Election. Exactly one device designates itself the leader and all others designate themselves follower. The computation ends when the leader sends a message while every follower listens to the channel.

Approximate Counting. At the end of the computation all devices agree on a number $\tilde{n}$ such that $\tilde{n}=\Theta(n)$.

Census. We only study this problem in the deterministic setting. At the end of the computation some device announces a list (or census) of the IDs of all devices.

REMARK 1. It could be argued that real-world devices rarely endow transmitters with more collision-detection power than receivers, so the Sender-CD model does not merit study. We feel this thinking gets the order backwards. There is a certain cost for equipping tiny devices with extra capabilities (such as generating random bits or detect collisions) so how are we to tell whether adding these capabilities is worth the expense? To answer that question we need to first determine the complexity of the algorithms that will ultimately run on the device. The goal of this work is to understand the power of various abstract models, not to cleave closely to existing real-world technologies, simply because they exist.

New Results. In the randomized model, we show that the energy complexity of Leader Election and Approximate Counting are $\Theta\left(\log ^{*} n\right)$ in Sender-CD and No-CD but $\Theta\left(\log \left(\log ^{*} n\right)\right)$ in Strong$\mathrm{CD}$ and Receiver-CD. The lower bounds also apply to the contention resolution problem, and this establishes that the recent $O\left(\log \left(\log ^{*} n\right)\right)$ contention resolution protocol of Bender et al. [7] is optimal. Our algorithm naturally offers a time-energy tradeoff. See Table 1 for the energy costs of our algorithm under different runtime constraints. For both upper bounds and lower bounds in the randomized model, the failure probability of an algorithm is at most $1 / \operatorname{poly}(n)$; and the devices in a failed execution may consume unbounded energy and never halt.

For Leader Election we establish matching bounds in all the deterministic models. In Strong-CD and Sender-CD, Leader Election and Census requires $\Omega(\log \log N)$ energy (even when $n=2$ ) and can be solved with $O(\log \log N)$ energy and $O(N)$ time, for any $n \leq N$. However, in No-CD and Receiver-CD, the complexity of these problems jumps to $\Theta(\log N)$ [24]. Meanwhile, Leader Election and Census can be solved with $\Theta(\log N)$ energy in the Receiver-CD and No-CD models.

Finally, we prove that when the input is dense in the ID space, meaning $n=\Theta(N)$, Census can actually be computed with only $O(\alpha(N))$ energy and $O(N)$ time, even in No-CD. To our knowledge, this is the first time inverse-Ackermann-type recursion has appeared in distributed computing.

\subsection{Prior Work}

Jurdzinski et al. [22] studied the deterministic energy complexity of Leader Election in the Sender-CD model. They proved that dense instances $(n=\Theta(N))$ can be solved with $O\left(\log ^{*} N\right)$ energy, and claimed that the complexity of the sparse problem is between $\Omega(\log \log N / \log \log \log N)$ and $O\left(\log ^{\epsilon} N\right)$. While the lower bound is correct, the algorithm presented in [22] is not. ${ }^{1}$ The most efficient published algorithm uses $O(\sqrt{\log N})$ energy, also due to Jurdzinski et al. [25]. The same authors [23] gave a reduction from randomized Sender-CD Approximate Counting to deterministic Leader Election over ID space $N=O(\log n)$, which, using [25], leads to an $O(\sqrt{\log \log n})$ energy algorithm for Approximate Counting. In [24] the authors gave a method for simulating Sender-CD protocols in the No-CD model, and proved that deterministic No-CD Leader Election takes $\Omega(\log N)$ energy. Nakano and Olariu [35] showed that $n$ devices in the Sender-CD model can assign themselves distinct IDs in $\{1, \ldots, n\}$ with $O(\log \log n)$ energy.

Recently Bender et al. [7] gave a method for circuit-simulation in the Strong-CD model, which led to randomized Approximate Counting and Leader Election protocols using $O\left(\log \left(\log ^{*} n\right)\right)$ energy and $n^{o(1)}$ time. An earlier algorithm of Kardas et al. [27] solves Leader Election in the Strong-CD model in $O\left(\log ^{\epsilon} n\right)$ time using $O(\log \log \log n)$ energy, in expectation.

Most of the previous work in the radio network model has been concerned with time, not energy. Willard [41] proved that $O(\log \log n)$ time is necessary and sufficient for one device to successfully transmit in the Strong-CD model with constant probability; see [36] for tradeoffs between time and success probability. In the Sender-CD model this problem requires $\Theta\left(\log ^{2} n\right)$ time to solve, with probability $1-1 / \operatorname{poly}(n)[17,26,37]$. Greenberg and Winograd [21] proved that if all devices need to send a message, $\Theta\left(n \log _{n}(N)\right)$ time is necessary and sufficient in the deterministic Strong-CD model.

The related problems of broadcasting, leader election, and gossiping have been studied extensively in multi-hop radio networks, where the bounds typically depend on both the diameter and size of the network, whether it is directed, and whether randomization and collision detection are available. See, e.g., $[1-3,10,11,13,14,18,30-$ 32]. Schneider and Watterhofer [39] investigated the use of collision detection in multihop radio networks when solving archetypal problems such as MIS, $(\Delta+1)$-coloring, and broadcast. Their results showed that the value of collision detection depends on the problem being solved.

Cornejo and Kuhn [12] introduced the beeping model, where no messages are sent; the only signals are $\left\{\lambda_{N}, \lambda_{S}\right\}$ : noise and silence.

${ }^{1} \mathrm{~T}$. Jurdzinski. (Personal communication, 2016.) 
Table 1: Time-energy tradeoff of randomized Approximate Counting and Leader Election.

\begin{tabular}{|l|l|l|}
\hline \multirow{2}{*}{ Time СомpLEXity } & \multicolumn{2}{|c|}{$\begin{array}{c}\text { ENERgy CompleXITY } \\
\text { Sender-CD or No-CD with } n>1\end{array}$} \\
\hline$O\left(n^{o(1)}\right)$ & Strong-CD or Receiver-CD & $O\left(\log ^{*} n\right)$ \\
\hline$O\left(\log ^{2+\epsilon} n\right), 0<\epsilon \leq O(1)$ & $O\left(\log \left(\log ^{*} n\right)\right)$ & $O\left(\epsilon^{-1} \log \log \log n\right)$ \\
\hline$O\left(\log ^{2} n\right)$ & $O\left(\log \left(\epsilon^{-1} \log \log \log n\right)\right)$ & $O(\log \log n)$ \\
\hline
\end{tabular}

The complexity of Approximate Counting was studied in [8] and the state-complexity of Leader Election was studied in [20].

In adversarial settings a jammer can interfere with communication. See $[15,33]$ for leader election protocols resilient to jamming. In a resource-competitive protocol [6] the energy cost of the devices is some function of the energy cost of the jammer. See [5] for resource-competitive contention resolution, and [19, 29] for resource-competitive point-to-point communication and broadcast protocols.

\subsection{Organization and Technical Overview}

To establish the two sharp exponential separations we need 8 distinct upper and lower bounds. The $O(\log N)$ upper bound on deterministic No-CD Leader Election is trivial and the matching lower bound in Receiver-CD is from [24]. The $O\left(\log \left(\log ^{*} n\right)\right)$ upper bound from [7] on randomized Leader Election/Approximate Counting works only in Strong-CD. This paper contains matching upper and lower bounds of $\Theta(\log \log N)$ in the deterministic Sender$\mathrm{CD} /$ Strong-CD models, and matching $\Theta\left(\log ^{*} n\right)$ bounds in the randomized No-CD/Sender-CD models. We adapt the randomized $O\left(\log \left(\log ^{*} n\right)\right)$ upper bound [7] from Strong-CD to Receiver-CD, and provide a matching $\Omega(\log (\log * n))$ lower bound in Strong-CD. In addition, we give a simpler proof of the $\Omega(\log N)$ lower bound in deterministic Receiver-CD.

In Section 2 we begin with a surprisingly simple proof that protocols solving any non-trivial problem in the deterministic StrongCD model require $\Omega(\log \log N)$ energy if the devices are adaptive and $\Omega(\log N)$ if they are non-adaptive. It turns out that ReceiverCD algorithms are essentially forced to be non-adaptive, so this yields $\Omega(\log N)$ lower bounds for deterministic Leader Election in Receiver-CD. The $\Omega(\log \log N)$ lower bound combines a decisiontree representation of the algorithm with the encoding argument that Katona and Szemerédi [28] used to solve the biclique covering problem of Erdős, Rényi, and Sós [16].

In Section 3 we prove the $\Omega\left(\log ^{*} n\right)$ and $\Omega\left(\log \left(\log ^{*} n\right)\right)$ lower bounds on randomized Approximate Counting/Leader Election. These lower bounds begin by embedding any algorithm into an infinite universal decision-DAG (basically a decision tree with some reconvergent paths). In this model two algorithms only differ in their transition probabilities and halting criteria. The proof is information-theoretic. We consider the only two methods for devices in Strong-CD/Receiver-CD to learn new information (i) via direct communication (in which one device transmits and some subset of devices listen) and (ii) via inference (in which transmitting/listening devices detect a collision or silence, which informs their future decisions). The information-theoretic capacity of method (i) is essentially unbounded whereas method (ii) is bounded by 1-bit per unit energy in Strong-CD and usually less in ReceiverCD. We show that any algorithm with a reasonable time bound can be forced to learn an approximation of $n$ via the informationtheoretically well-behaved method (ii).

In Section 4 we present the deterministic upper bound. We devise an $O(\log \log N)$-energy protocol for Leader Election and Census. The protocol combines van Emde Boas-like recursion with a technique that lets a group of devices function as one device and thereby share energy costs. In the full version of the paper [9], we present an $O(\alpha(N))$ energy protocol for dense Leader Election/Census, when $n=\Theta(N)$.

In the full version of the paper [9], we present upper bounds on randomized Leader Election/Approximate Counting. When time is not too constrained (suppose the budget is $n^{o(1)}$ ), the Sender-CD and Receiver-CD protocols have energy complexity $O\left(\log ^{*} n\right)$ and $O\left(\log \left(\log ^{*} n\right)\right)$. Our protocols naturally adapt to any time bound that is $\Omega\left(\log ^{2} n\right)$, where the energy complexity gradually increases as we approach this lower limit. See Table 1. These protocols are randomized (and do not assume distinct IDs); nonetheless, they use the deterministic $\alpha(N)$ dense Leader Election/Census algorithm.

\section{DETERMINISTIC LOWER BOUNDS}

In this section we prove deterministic lower bounds on the Successful Communication problem, which is no harder than Leader Election. The goal is to have some time slot where exactly one device sends a message while at least one device listens. Once successful communication occurs the algorithm is terminated on all devices. We focus on the special case when $n=2$, both devices know $n=2$, but not the ID of the other device. Since Strong-CD and Sender-CD models are the same when $n=2$, our lower bound applies to both.

We first address the case of non-adaptive algorithms, where the actions of a device are solely a function of its ID, not the signals it receives by transmitting/listening to the channel. We then extend the proof technique to the more general adaptive case.

THEOREM 2.1. The energy cost of a non-adaptive Leader Election algorithm is $\Omega(\log N)$, even when $n=2$.

Proof. Let $\tau=\tau(N)$ be the running time of a non-adaptive algorithm for Successful Communication. This algorithm can be encoded by a table in the set $\{T, L, I\}^{\tau \times N}$; see Figure 1a. The $(j, i)$-th entry of the table is the action (transmit, listen, or idle) made by the device with ID $i$ at time $j$. The energy cost of device $i$, denoted $E_{i}$, is the number of $T$ or $L$ entries in the $i$ th column. The two active devices must successfully communicate in some time slot. If their IDs are $\alpha$ and $\beta$, there must be a row in the table where the entries of the $\alpha$ th and the $\beta$ th columns contain one $T$ and one $L$.

We now prove that $\max _{i} E_{i} \geq \log N$. The proof is inspired by Katona and Szemerédi's [28] lower bound of the biclique covering 


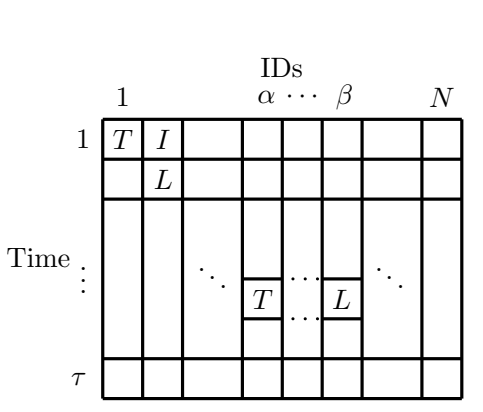

(a)
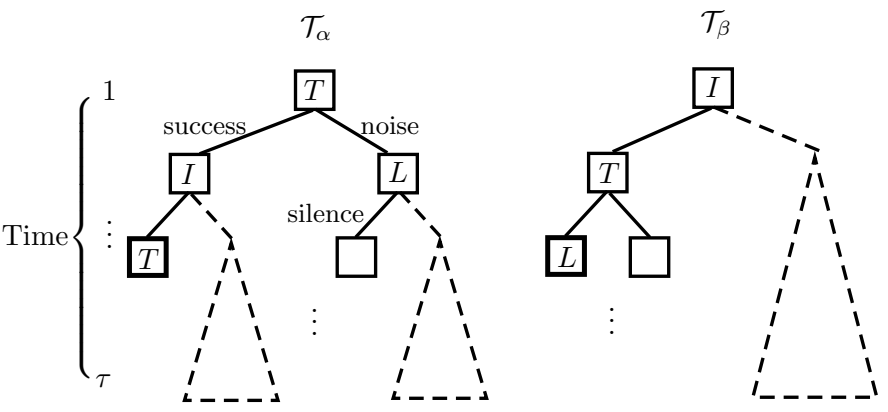

(b)

Figure 1: (a) The non-adaptive action table. Any two columns must have a corresponded pair of $T$ and $L$. (b) Two binary decision trees $\mathcal{T}_{\alpha}$ and $\mathcal{T}_{\beta}$ in the adaptive case. There is a corresponded pair of $T$ and $L$ in the two trees with the same left-right path from the roots.

problem. Encode the $i$ th column as a binary string of length $\tau$ by replacing $T$ with $0, L$ with 1 , and $I$ with either 0 or 1 . There are clearly $2^{\tau-E_{i}}$ possible encodings for column $i$. Since every pair of columns must have a row where they contain $T$ and $L$, no binary string is an eligible encoding of two distinct columns. Since there are $2^{\tau}$ possible encodings, we have:

$$
\sum_{i=1}^{N} 2^{\tau-E_{i}} \leq 2^{\tau}, \quad \text { which implies that } \sum_{i=1}^{N} \frac{1}{2^{E_{i}}} \leq 1 .
$$

The convexity of $f(x)=1 / 2^{x}$ implies $\sum_{i} E_{i} \geq N \log N$. So the energy cost of the algorithm is $\Omega(\log N)$, even on average.

Theorem 2.2. The energy cost of a deterministic Leader Election is $\Omega(\log \log N)$ in the Strong-CD and Sender-CD models, even when $n=2$.

Proof. Suppose we have an algorithm for Successful Communication running in $\tau$ time. Fixing $n=2$, we represent the behavior of the algorithm on the device with ID $i$ as a binary decision tree $\mathcal{T}_{i}$. Each node in $\mathcal{T}_{i}$ is labeled with an action in $\{T, L, I\}$. An $I$ (idle) node has one left child and no right child; a $T$ (transmit) node has two children, a left one indicating collision-free transmisssion and a right one indicating a collision. An $L$ node has two children, a left one indicating silence and a right one indicating the transmission of some message (and the termination of the algorithm).

The left-right ordering of children is meaningless but essential to making the following arguments work. Suppose that we run the algorithm on devices with IDs $\alpha$ and $\beta$, and halt the algorithm at the first time $t$ in which successful communication occurs. We claim the paths taken through $\mathcal{T}_{\alpha}$ and $\mathcal{T}_{\beta}$ are encoded by the same sequence of $t-1$ left/right turns. At any time slot before $t$ the possible actions performed by $\{\alpha, \beta\}$ are $\{I, I\},\{I, T\},\{I, L\},\{L, L\},\{T, T\}$. In all cases, both $\alpha$ and $\beta$ branch to the left, except for $\{T, T\}$, in which case they both branch right. At time $t$ the actions are $\{T, L\}$ and it is only here that they branch in opposite directions. See Figure $1 \mathrm{~b}$.

To encode $\mathcal{T}_{i}$ as a bit-string we extend it to a full binary tree of depth $\tau$ : add a dummy right child to every $I$-node, and repeatedly add two dummy children to any leaf at depth less than $\tau$. The right child of an $L$-node is considered a dummy. The number of nodes in $\mathcal{T}_{i}$ is now $2^{\tau}-1$. We encode $\mathcal{T}_{i}$ as a bit-string of length $2^{\tau}-1$ by listing the nodes in any fixed order (say preorder), mapping each $T$-node to $0, L$-node to 1 , and each $I$-node or dummy node to either 0 or 1 . Since any $\alpha, \beta$ must engage in successful communication, there must be a position in the full binary tree that is a $T$-node in $\mathcal{T}_{\alpha}$ and an $L$-node in $\mathcal{T}_{\beta}$ or vice versa. Hence no bit-string is an eligible encoding of two distinct $\mathcal{T}_{\alpha}, \mathcal{T}_{\beta}$. If device $i$ spends energy $E_{i}$, then the number of $T$ or $L$ nodes in $\mathcal{T}_{i}$ is at most $2^{E_{i}}-1$ and therefore $\mathcal{T}_{i}$ has $2^{\left(2^{\tau}-1\right)-\left(2^{E_{i}}-1\right)}$ possible encodings. Thus,

$\sum_{i=1}^{N} 2^{\left(2^{\tau}-1\right)-\left(2^{E_{i}}-1\right)} \leq 2^{2^{\tau}-1}$ which implies that $\sum_{i=1}^{N} \frac{1}{2^{2^{E_{i}}-1}} \leq 1$.

The convexity of $f(x)=1 / 2^{2^{x}-1}$ implies $\sum_{i} E_{i} \geq N \log (\log N+$ 1 ), so the energy cost of the algorithm is $\Omega(\log \log N)$, even on average.

Notice that in the decision trees above, only transmitting nodes are able to branch before the algorithm halts with a successful communication. In the No-CD model, however, transmitters receives no feedback. In this case, no branching is possible before the first successful communication and the problem reduces to the nonadaptive case. Therefore we also obtain an $\Omega(\log N)$ lower bound for Leader Election in the No-CD model, which matches the trivial upper bound, and an earlier lower bound of [24]. The lower bound also applies to the stronger Receiver-CD model since when $n=2$, the Receiver-CD model is the same as No-CD.

Corollary 2.3. The energy cost of Leader Election is $\Theta(\log N)$ in the No-CD and Receiver-CD models, even when $n=2$.

\section{RANDOMIZED LOWER BOUNDS}

In this section we prove energy lower bounds of randomized algorithms for Approximate Counting. Our lower bounds hold even in the scenario where the devices in a failed execution may consume unbounded energy and never halt. 
THEOREM 3.1. The energy cost of a polynomial time Approximate Counting algorithm with failure probability $1 / n$ is $\Omega\left(\log ^{*} n\right)$ in the Sender-CD model.

Theorem 3.2. The energy cost of a polynomial time Approximate Counting algorithm with failure probability $1 / n$ is $\Omega\left(\log \left(\log ^{*} n\right)\right)$ in the Strong-CD model.

Since No-CD is strictly weaker than Sender-CD, the $\Omega\left(\log ^{*} n\right)$ lower bound also applies to No-CD. Similarly, the $\Omega\left(\log \left(\log ^{*} n\right)\right)$ lower bound applies to Receiver-CD.

\subsection{Randomized Decision Trees}

The process of a device $s$ interacting with the network at time slot $t$ has two phases. During the first phase (action performing phase), $s$ decides on its action, and if this action is to transmit, then $s$ chooses a message $m \in \mathcal{M}$ and transmits $m$. During the second phase (message receiving phase), if $s$ chose to listen or transmit during the first phase, then $s$ hears the signal (depending on the model) which depends on the transmissions occurring at this time slot. The phases partition the timeline into layers. We write layer $t$ to denote the time right before the first phase of time slot $t$, and layer $t+0.5$ to denote the time right before the second phase of time slot $t$. The choice of the message space $\mathcal{M}$ is irrelevant to our lower bound proof. The cardinality of $\mathcal{M}$ may be finite or infinite.

For a device $s$, the state of $s$ at layer $t$ includes the ordered list of actions taken by $s$ and signals received from the channel until layer $t$. Layer 1 consists of only the initial state, which is the common state of all devices before the execution of an algorithm.

Our lower bounds are proved using a single decision tree $\mathcal{T}$ of unbounded branching factor (if $|\mathcal{M}|$ is unbounded). A special directed acyclic graph (DAG) $\mathcal{G}$ is defined to capture the behaviour of any randomized algorithm, and then the decision tree $\mathcal{T}$ is constructed by "short-cutting" some paths in $\mathcal{G}$.

The $D A G \mathcal{G}$. The set of all nodes in $\mathcal{G}$ represent all possible states of a device during the execution of any algorithm. Similarly, the set of all arcs represent all legal transitions between states during the execution of any algorithm. Therefore each arc connects only nodes in adjacent layers, and the root of $\mathcal{G}$ is the initial state.

Let $t \in \mathbb{Z}^{+}$. A transition from a state $u$ in layer $t$ to a state $v$ in layer $t+0.5$ corresponds to one of the possible $|\mathcal{M}|+2$ actions that can be performed in the first phase of time slot $t$. The transitions from a state $u$ in layer $t+0.5$ to a state $v$ in layer $t+1$ are more involved. Based on the action performed in the first phase of time slot $t$ that leads to the state $u$, there are three cases:

- If the action is idle, then $u$ has one outgoing arc corresponding to doing nothing.

- If the action is listen, then $u$ has $|\mathcal{M}|+2$ outgoing arcs in the Strong-CD model (or $|\mathcal{M}|+1$ in the Sender-CD model), corresponding to all possible messages that can be heard.

- If the action is transmit, then $u$ has two outgoing arcs. The first (second) outgoing arc corresponds to the message transmission succeeding (failing). If a failure took place, then no other device knows which message was sent by the device, and so the content of this message is irrelevant. Thus, all states $u$ in layer $t+0.5$ that correspond to the action transmit and share the same parent have the same child node in layer $t+1$ corresponding to a failure in transmitting the message. Notice that the arcs corresponding to failed transmissions are what make $\mathcal{G}$ a DAG and not a tree.

Embedding an Algorithm. Any algorithm can be embedded into $\mathcal{G}$. An algorithm $\mathcal{A}$ is embedded into $\mathcal{G}$ as follows. First of all, appropriate states, depending on $\mathcal{A}$, are designated as terminal states. Without loss of generality, we require that any terminal state must be in layer $t$ for some $t \in \mathbb{Z}^{+}$. Each terminal state is associated with a specific output for the problem at hand. A device entering a terminal state $u$ terminates with the output associated with the state $u$. Any randomized algorithm is completely described by designating the terminal states together with their outputs, and specifying the transition probabilities from states in layer $t$ to states in layer $t+0.5$ for all $t \in \mathbb{Z}^{+}$. We require that the transition probabilities of all transitions from a given state in layer $t$ sum up to 1.

Randomized Decision Tree $\mathcal{T}$. The tree $\mathcal{T}$ is derived from $\mathcal{G}$ as follows. The set of nodes of $\mathcal{T}$ is the set of nodes in $\mathcal{G}$ that are in layer $t$ such that $t \in \mathbb{Z}^{+}$. For any two states $u$ in layer $t \in \mathbb{Z}^{+}$and $v$ in layer $t+1$ that are linked by a directed path, there is a transition from $u$ to $v$ in $\mathcal{T}$. It is straightforward to see that $\mathcal{T}$ is a rooted tree. See Figure 2 for an illustration of both $\mathcal{G}$ and $\mathcal{T}$ in the Strong-CD model with $\mathcal{M}=\left\{m_{1}, \ldots, m_{k}\right\} .^{2}$ For a state $u$ in layer $t \in \mathbb{Z}^{+}$, and for an action $x \in\{$ idle, listen, transmit $\}$, we write $p_{u \rightsquigarrow x}$ to denote the probability that a device belonging to $u$ chooses to do the action $x$ in the first phase of time slot $t$.

Time and Energy Complexity. An execution of an algorithm for a device is completely described by a directed path $\left(u_{1}, u_{2}, \ldots, u_{k}\right)$ in $\mathcal{T}$ such that $u_{t}$ is in time slot $t$ for each $1 \leq t \leq k$, and $u_{k}$ is the only terminal state. The runtime of the device is $k$. The amount of energy the device spends is the number of transitions corresponding to listen or transmit in $\left(u_{1}, u_{2}, \ldots, u_{k}\right)$. The time (energy) of an execution of an algorithm is the maximum time (energy) spent by any device.

\subsection{Lower Bound in the Sender-CD Model}

Let $\mathcal{A}$ be any $T(n)$ time algorithm for Approximate Counting in the Sender-CD model with failure probability at most $1 / n$. We assume that $T(n) \geq n$ throughout the section. ${ }^{3}$ An energy lower bound of $\mathcal{A}$ is shown by carefully selecting a sequence of network sizes $\left\{n_{i}\right\}$ with checkpoints $\left\{d_{i}\right\}$ such that $d_{i}<n_{i} \leq T\left(n_{i}\right)<d_{i+1}$.

There are two main components in the lower bound proof. The first component is to show that, with probability $1-1 / \operatorname{poly}\left(n_{i}\right)$, no message is successfully transmitted before time $d_{i}$ when running $\mathcal{A}$ on $n_{i}$ devices (that is, every transmission ends in a collision). This limits the amount of information that could be learned from a device, as the only thing a device can learn at a given time slot is whether there are zero or at least two devices trying to transmit

\footnotetext{
${ }^{2}$ Notice that in the Strong-CD model, a device transmitting a message $m_{i}$ to the channel at a time slot must not hear $\lambda_{S}$ in the same time slot. If the transmission is successful, it hears the message $m_{i}$; otherwise it hears $\lambda_{N}$.

${ }^{3}$ The following simple fix increases the runtime of any Approximate Counting algorithm to $T(n) \geq n$ without affecting the energy cost. Suppose that the estimate $\tilde{n}$ all devices agree on is within a multiplicative factor $c \geq 1$ of $n$. After a device decides its estimate $\tilde{n}$, let it remains idle for $c \cdot \tilde{n}$ additional time slots before it terminates.
} 

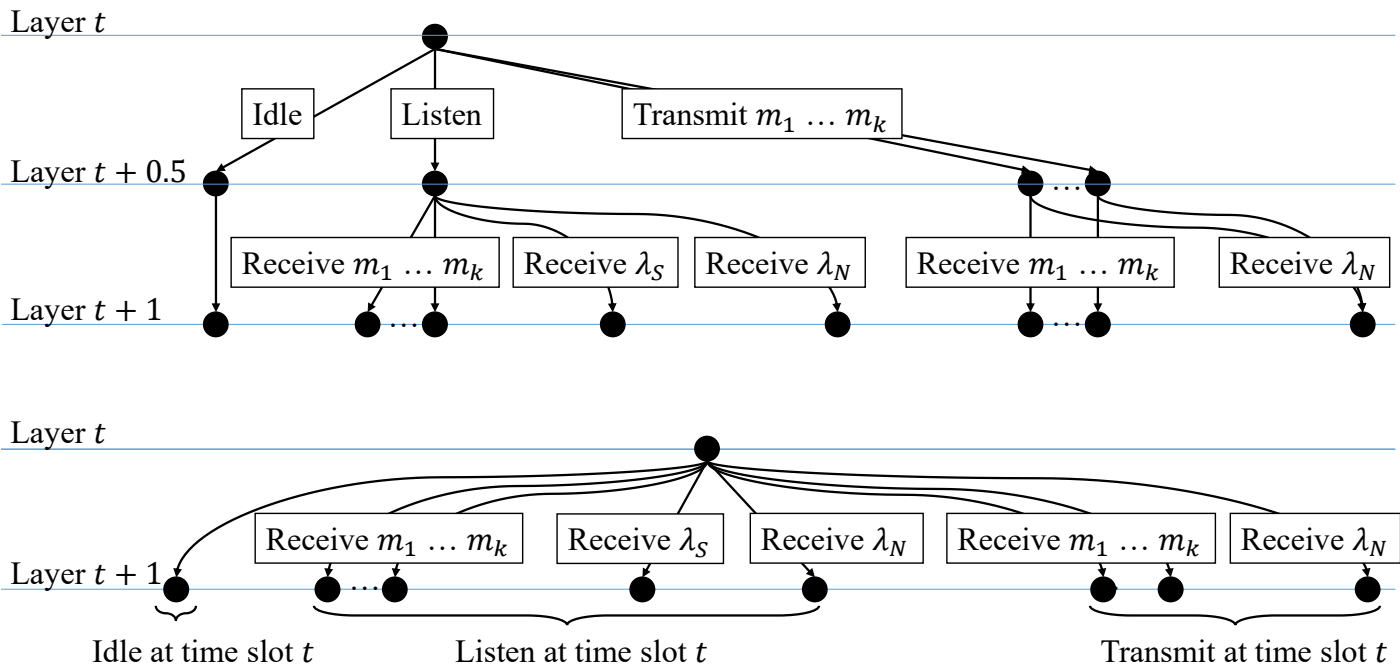

Figure 2: Upper: a portion of $\mathcal{G}$. Lower: the corresponding portion in $\mathcal{T}$.

some message to the channel. The second component is to prove that, for $j>i$, in order for a device to have enough information to distinguish between $n_{i}$ and $n_{j}$ within $T\left(n_{i}\right)<d_{i+1}$ time slots, that device must use at least one unit of energy within time interval $\left[d_{i}, d_{i+1}-1\right]$. Intuitively, this is because a device only gains information from either listen or transmit.

Truncated Decision Tree. The no-communication tree $\mathcal{T}_{\text {no-comm }}$ is defined as the subtree of $\mathcal{T}$ induced by the set of all states $u$ such that no transition in the path from the root to $u$ corresponds to receiving a message in $\mathcal{M}$. In other words, $\mathcal{T}_{\text {no-comm }}$ contains exactly the states whose execution history contains no successful communication. Notice that in Sender-CD each state in $\mathcal{T}_{\text {no-comm }}$ has exactly three children, and the three children correspond to the following three pairs of action performed and message received: (transmit, $\lambda_{S}$ ), (listen, $\lambda_{S}$ ), and (idle, N/A). For each state $u$ at layer $t$ of the tree $\mathcal{T}_{\text {no-comm }}$, we define the probability estimate $p_{u}$ inductively as follows. If $u$ is the root, $p_{u}=1$; otherwise $p_{u}=p_{v} \cdot p_{v \rightsquigarrow x}$, where $v$ is the parent of $u$, and $x$ is the action performed at time slot $t-1$ that leads to the state $u$. Intuitively, if no message is successfully sent in an execution of $\mathcal{A}$, the proportion of devices entering $u$ is concentrated around $p_{u}$, given that $p_{u}$ is high enough See Figure 3 for an illustration of no-communication tree $\mathcal{T}_{\text {no-comm }}$ and probability estimates in the Sender-CD model.

Checkpoints $d_{i}$ and Network Sizes $n_{i}$. Given the runtime constraint $T(n)$, we define an infinite sequence of checkpoints as follows: $d_{1}$ is a large enough constant to be determined; for all $i>1$, $d_{i}=T\left(2^{2^{2^{2^{d_{i-1}}}}}\right)$.

LEMMA 3.3. For each index $i$, there exists a network size $n_{i}$ with $2^{2^{d_{i}}}<n_{i}<2^{2^{2^{d^{d_{i}}}}}$ such that for each state $u \in \mathcal{T}_{\text {no-comm }}$ at layer at most $d_{i}$, either $p_{u} \leq n_{i}{ }^{-10}$ or $p_{u} \geq n_{i}{ }^{-1 / 10}$.

Proof. Define $m_{1}=2^{2^{d_{i}}}+1$; for $1<k \leq 3^{d_{i}}$, define $m_{k}=$ $m_{k-1} 100+1$. It is straightforward to see that $2^{2^{d_{i}}}<m_{1}<m_{2}<$ $\ldots<m_{3} d_{i}<2^{2^{2^{d^{d}}}}$, as long as $d_{i}$ is at least a large enough constant. For each state $u \in \mathcal{T}_{\text {no-comm }}$ at layer at most $d_{i}$, there exists at most one $m_{k}$ with $m_{k}{ }^{-10}<p_{u}<m_{k}{ }^{-1 / 10}$. Recall that $\mathcal{T}_{\text {no-comm has }}$ branching factor 3 , and hence the number of states up to layer $d_{i}$ is less than $3^{d_{i}}$. By the pigeonhole principle, among the $3^{d_{i}}$ distinct integers $m_{1}, m_{2}, \ldots$, there exists one integer $n_{i}$ such that, for each state $u \in \mathcal{T}_{\text {no-comm }}$ at layer at most $d_{i}$, either $p_{u} \leq n_{i}{ }^{-10}$ or $p_{u} \geq n_{i}{ }^{-1 / 10}$.

For each index $i, n_{i}$ is chosen to meet the statement of Lemma 3.3, and the first checkpoint $d_{1}$ is chosen to be a large enough number such that $n_{i}$ and $n_{i+1}$ are not within a constant approximation. We define $\mathcal{T}_{i}$ as the subtree of $\mathcal{T}_{\text {no-comm }}$ that consists of all states $u$ up to layer $d_{i}$ such that $p_{u} \geq n_{i}{ }^{-1 / 10}$. Notice that $\mathcal{T}_{i} \subseteq \mathcal{T}_{i+1}$ for all $i$. For an execution of $\mathcal{A}$ on $n_{i}$ devices, we write $\mathcal{P}_{i}$ to denote the event where for each state $u$ in layer $1 \leq t \leq d_{i}$ of the decision tree $\mathcal{T}$, the number of devices entering $u$ is within $n_{i} \cdot p_{u} \pm t \cdot n_{i}{ }^{0.6}$ if $u$ is in layer $t$ of $\mathcal{T}_{i}$, and is 0 if $u \notin \mathcal{T}_{i}$.

Lemma 3.4. Let $t \leq d_{i}$, and let $v$ be a state in layer $t-1$ of $\mathcal{T}_{i}$ such that the number of devices entering $v$ is within $n_{i} \cdot p_{v} \pm(t-1) \cdot n_{i}{ }^{0.6}$, and let $x$ be an action in $\{$ transmit, listen, idle\}. With probability $1-O\left(n_{i}{ }^{-9}\right)$, the number of devices that are in state $v$ at time $t-1$ and perform action $x$ at time $t-1$ is (i) within $n_{i} \cdot p_{v} \cdot p_{v} \rightarrow x \pm t \cdot n_{i}{ }^{0.6}$ when $p_{v} \cdot p_{v \rightsquigarrow x} \geq n_{i}{ }^{-1 / 10}$, or is (ii) zero when $p_{v} \cdot p_{v \rightsquigarrow x} \leq n_{i}{ }^{-10}$.

Proof. Define $m$ as the number of devices that are in state $v$ at time $t-1$ and do action $x$ at time $t$. According to our choice of $n_{i}$, for each state $u \in \mathcal{T}_{\text {no-comm }}$ at layer at most $d_{i}$, either $p_{u} \leq n_{i}{ }^{-10}$ or $p_{u} \geq n_{i}{ }^{-1 / 10}$. Since $p_{v} \cdot p_{v \rightsquigarrow x}=p_{u}$ for some $u \in \mathcal{T}_{\text {no-comm }}$ at layer at most $d_{i}$, we have the following two cases:

- Case 1: $n_{i}{ }^{-10} \geq p_{v} \cdot p_{v \rightsquigarrow x}$. We have $p_{v \rightsquigarrow x} \leq n_{i}{ }^{-10} / p_{v} \leq$ $n_{i}{ }^{-9.9}$, and we have $\mathrm{E}[m] \leq\left(n_{i} \cdot p_{v}+(t-1) \cdot n_{i}{ }^{0.6}\right)$. $p_{v \rightsquigarrow x} \leq n_{i}{ }^{-9}+(t-1) \cdot n_{i}{ }^{0.6} \cdot p_{v \rightsquigarrow x} \leq n_{i}{ }^{-9}+(t-1) \cdot n_{i}{ }^{-9.3}<$ $2 n_{i}^{-9}$ (recall that $t \leq d_{i}<\log \log n_{i}$ ). By Markov's inequality, $m=0$ with probability $\geq 1-2 n_{i}{ }^{-9}$. 


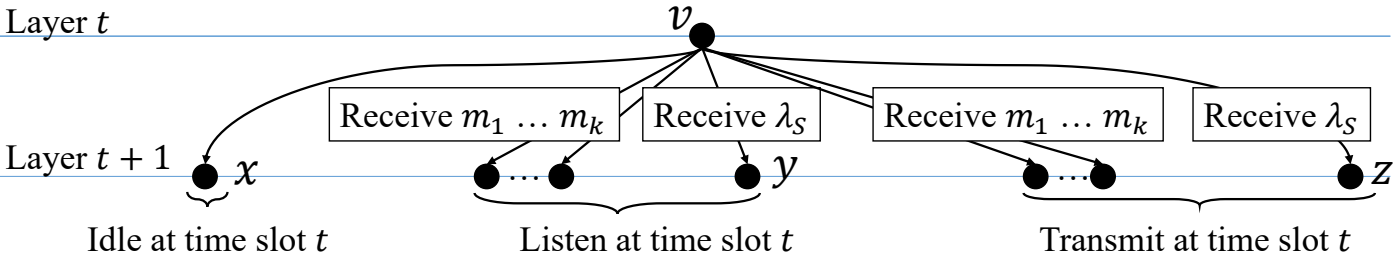

Idle at time slot $t$

Listen at time slot $t$

Transmit at time slot $t$

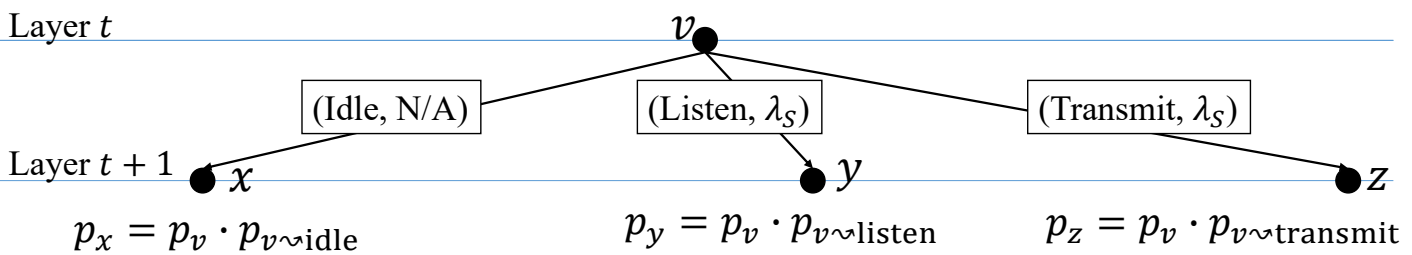

Figure 3: Upper: a portion of the tree $\mathcal{T}$. Lower: the corresponding portion in the no-communication tree $\mathcal{T}_{\text {no-comm }}$

- Case 2: $p_{v} \cdot p_{v} \rightsquigarrow x \geq n_{i}{ }^{-1 / 10}$. The value of $\mathrm{E}[m]$ is within $p_{v \rightsquigarrow x} \cdot\left(n_{i} \cdot p_{v} \pm(t-1) \cdot n_{i}^{0.6}\right)$, which is within the range $n_{i} \cdot p_{v} \cdot p_{v \rightsquigarrow x} \pm(t-1) \cdot n_{i}{ }^{0.6}$. Let $\delta=n_{i}{ }^{-0.4} / 2$. Notice that we have $\delta \cdot \mathrm{E}[m]<n_{i}{ }^{0.6}$ and $\mathrm{E}[m]>n_{i}{ }^{0.9} / 2$. Meanwhile, each device in the state $v$ decides which action to perform next independently. By Chernoff bound, the probability that $m$ is within $1 \pm \delta$ fraction of $\mathrm{E}[\mathrm{m}]$ is at least $1-2 \exp \left(-\delta^{2}\right.$. $\mathrm{E}[m] / 3) \geq 1-2 \exp \left(-\left(n_{i}{ }^{-0.4} / 2\right)^{2} \cdot\left(n_{i}{ }^{0.9} / 2\right) / 3\right)>1-$ $O\left(n_{i}{ }^{-9}\right)$. Therefore, with such probability, $m$ is in the range $\mathrm{E}[m](1 \pm \delta)$, which is within $n_{i} \cdot p_{v} \cdot p_{v \rightsquigarrow x} \pm t \cdot n_{i}{ }^{0.6}$.

\section{$\square$}

Lemma 3.5. For an execution of $\mathcal{A}$ on $n_{i}$ devices, $\mathcal{P}_{i}$ holds with probability at least $1-n_{i}{ }^{-7}$.

Proof. For the base case of $t=1$, it is straightforward to see that $\mathcal{P}_{i}$ holds for the initial state. For each $1<t \leq d_{i}$, assuming $\mathcal{P}_{i}$ holds for all states at layer $t-1$, then we show that $\mathcal{P}_{i}$ holds for all states at layer $t$ with probability at least $1-O\left(n_{i}{ }^{-8}\right)$.

That $\mathcal{P}_{i}$ holds for all states at layer $t-1$ guarantees that for each state $v$ in layer $t-1$ of $\mathcal{T}_{i}$, the number of devices entering $v$ is within $n_{i} \cdot p_{v} \pm(t-1) \cdot n_{i}{ }^{0.6}$. By the union bound on at most $3^{t-1}$ choices of $v$ and 3 choices of $x$, the statement of Lemma 3.4 holds for all choices of $v$ and $x$ with probability at least $1-\left(3^{t}\right) \cdot O\left(n_{i}{ }^{-9}\right) \geq 1-O\left(n_{i}{ }^{-8}\right)$ (recall that $t<\log \log n_{i}$ ). This implies that the number of devices transmitting at time $t-1$ is either 0 or at least $n_{i}{ }^{0.9}-t \cdot n_{i}{ }^{0.6}>1$, and hence no message is successfully sent by the layer $t$ (i.e. right before the time slot $t$ ). Therefore, at layer $t$, all devices are confined in states within $\mathcal{T}_{\text {no-comm }}$. Let $u$ be the child of $v$ in $\mathcal{T}_{\text {no-comm }}$ such that the action leading to $u$ is $x$. By the choice of $n_{i}, u \in \mathcal{T}_{i}$ implies that $p_{u}=p_{v} \cdot p_{v \rightsquigarrow x} \geq n_{i}{ }^{-1 / 10}$; and $u \notin \mathcal{T}_{i}$ implies that $p_{u}=$ $p_{v} \cdot p_{v \rightsquigarrow x} \leq n_{i}{ }^{-10}$. Therefore, assuming $\mathcal{P}_{i}$ holds for all states at layer $t-1$, Lemma 3.4 guarantees that, with probability at least $1-O\left(n_{i}{ }^{-8}\right), \mathcal{P}_{t}$ holds for all states at layer $t$.

By the union bound on all $t \in\left\{1, \ldots, d_{i}\right\}, \mathcal{P}_{i}$ holds with probability at least $1-n_{i}{ }^{-7}$. Notice that $d_{i}<\log \log n_{i}$.

We claim that $\mathcal{T}_{\text {no-comm }}$ has no terminal state $u$ with $p_{u} \neq 0$. Suppose that $u \in \mathcal{T}_{\text {no-comm }}$ is a terminal state with $p_{u} \neq 0$. Then there exists an index $i$ such that for all $j \geq i, u \in \mathcal{T}_{j}$. Among all $\left\{n_{j}\right\}_{j \geq i}$, the decision of $u$ is a correct estimate of at most one $n_{j}$ (recall that any two network sizes in $\left\{n_{j}\right\}_{j \geq 1}$ are not within a constant factor of each other). Therefore, the adversary can choose one network size $n_{j^{\prime}}$ from $\left\{n_{j}\right\}_{j \geq i}$ such that when $\mathcal{A}$ is executed on $n_{j^{\prime}}$ devices, any device entering $u$ gives a wrong estimate of $n_{j^{\prime}}$. By Lemma 3.5, with probability $1-n_{j^{\prime}}{ }^{-7}>1 / n_{j^{\prime}}$, there is a device entering $u$, and hence the algorithm fails with probability higher than $1 / n_{j^{\prime}}$, a contradiction.

Forcing Energy Expenditure. Let $p_{\text {idle }}$ be the probability for a device entering a state $u$ in layer $d_{i}$ of $\mathcal{T}_{i}$ to be idle throughout the time interval $\left[d_{i}, d_{i+1}-1\right]$. For a device $s$ to correctly give an estimate of network size $n_{i}$ within the time constraint $T\left(n_{i}\right)<d_{i+1}$, the device $s$ must successfully hear some message $m \in \mathcal{M}$. Recall that $\mathcal{T}_{\text {no-comm has no terminal state } u \text { with }} p_{u} \neq 0$, and hence a device must leave the tree $\mathcal{T}_{\text {no-comm }}$ before it terminates. If $\mathcal{P}_{i}$ holds, then one unit of energy expenditure in the time interval $\left[d_{i}, d_{i+1}-1\right]$ is required for any device $s$ in a state in layer $d_{i}$ of $\mathcal{T}_{i}$. Therefore, for all devices to terminate by time $T\left(n_{i}\right)$ with probability at least $1-1 / n_{i}$ when $\mathcal{A}$ is executed on $n_{i}$ devices, we need $1 / n_{i} \geq \operatorname{Pr}\left[\mathcal{P}_{i}\right] \cdot p_{\text {idle, }}$, and this implies $p_{\text {idle }}<2 / n_{i} \leq 2 \cdot 2^{-2^{d_{i}}}<2^{-d_{i}}$. In other words, for any device in a state in layer $d_{i}$ of $\mathcal{T}_{i}$, the device spends at least one unit of energy in the time interval $\left[d_{i}, d_{i+1}-1\right]$ with probability at least $1-p_{\text {idle }}>1-2^{-d_{i}}$.

The Lower Bound. Consider an execution of $\mathcal{A}$ on $n_{i}$ devices, and let $s$ be any one of the $n_{i}$ devices. Let $j \in\{1, \ldots, i\}$. We claim that, given that $\mathcal{P}_{i}$ holds, with probability $1-2 \cdot 2^{-d_{j}}$ the device $s$ spends at least one unit of energy in the interval $\left[d_{j}, d_{j+1}-1\right]$.

- First, we show that the probability that $s$ enters a state in layer $d_{j}$ of $\mathcal{T}_{j}$ is at least $1-2^{-d_{j}}$. Recall that $\mathcal{T}_{j}$ is a subtree of $\mathcal{T}_{i}$. By definition, if $u$ is a state in layer $d_{j}$ but not belongs to $\mathcal{T}_{j}$, then $p_{u}<n_{j}{ }^{-1 / 10}$. Therefore, the proportion of the devices that do not enter a state in layer $d_{j}$ of $\mathcal{T}_{j}$ is at most $\frac{1}{n_{i}}\left(n_{i} \cdot n_{j}{ }^{-1 / 10}+d_{j} \cdot n_{i}{ }^{0.6}\right) \cdot 3^{d_{j}}=$ $\left(n_{j}{ }^{-1 / 10}+d_{j} \cdot n_{i}{ }^{-0.4}\right) \cdot 3^{d_{j}}<2^{-d_{j}}$. 
- Second, as argued in the above paragraph, a device that enters a state in layer $d_{j}$ of $\mathcal{T}_{j}$ spends at least one unit of energy in the time interval $\left[d_{j}, d_{j+1}-1\right]$ with probability $1-2^{-d_{j}}$.

Therefore, the claim is concluded.

By the union bound on $j=1, \ldots, i$, the probability that the device $s$ spends at least one unit of energy in each of the intervals $\left[d_{j}, d_{j+1}-1\right], 1 \leq j \leq i$, is at least $\operatorname{Pr}\left[\mathcal{P}_{i}\right]-2 \sum_{j=1}^{i} 2^{-d_{j}}>1 / 2$ (we choose $d_{1}$ to be a large enough number to make the inequality hold). We conclude the theorem.

THEOREM 3.6. For any $i \geq 1$, there exists a network size $n$ with $d_{i}+1 \leq n \leq d_{i+1}$ such that if $\mathcal{A}$ is executed on $n$ devices, for any device $s$, with probability at least $1 / 2$ the device $s$ spends at least one unit of energy in each of the time intervals $\left[d_{j}, \ldots, d_{j+1}-1\right]$, $1 \leq j \leq i$.

$\overbrace{2_{2^{2^{n}}}^{\alpha}}^{\alpha}$

As long as $T(n)=O\left(2^{2^{2^{2^{n}}}}\right)$ for a constant $\alpha$, the energy cost of Approximate Counting in the Sender-CD model is $\Omega\left(\log ^{*} n\right)$. Our lower bound proof naturally offers a time-energy tradeoff. For example, the lower bound generalizes to higher time constraints as follows. For any function $g(n) \leq \log n$, under time constraint $T(n)=g^{-1}(n)$, the energy cost is $\Omega\left(g^{*}(n)\right)$.

\subsection{Lower Bound in the Strong-CD Model}

Let $\mathcal{A}$ be any $T(n)$ time algorithm for Approximate Counting in the Strong-CD model with failure probability at most $1 / n$. Similar to the Section 3.2, we construct a sequence of network sizes $\left\{n_{i}\right\}$ with checkpoints $\left\{d_{i}\right\}$ such $d_{i}<n_{i} \leq T\left(n_{i}\right)<d_{i+1}$. Each pair $\left(n_{i}, d_{i}\right)$ is associated with a truncated decision tree $\mathcal{T}_{i}$ such that with probability $1-1 / \operatorname{poly}\left(n_{i}\right)$ the execution history of all devices are confined in $\mathcal{T}_{i}$ when running $\mathcal{A}$ on $n_{i}$ devices by time $d_{i}$. Due to the capability of differentiating between silence and noise in the Strong-CD model, a device is able to perform a binary search on a set of candidate network sizes $\left\{n_{i}\right\}_{1 \leq i \leq k}$, which costs only $O(\log k)$ unit of energy. In comparison, in the Sender-CD model $O(k)$ energy consumption is needed in the worst case. In the lower bound proof, we construct a path $P$ in $\mathcal{T}_{\text {no-comm }}$ which captures the worst case scenario of the binary search, and we will see that the energy consumption of a device whose execution history follows the path $P$ is $\Omega\left(\log \left(\log ^{*} n\right)\right)$.

Checkpoints $d_{i}$, Network Sizes $n_{i}$, and Subtrees $\mathcal{T}_{i}$. The definitions of no-communication tree $\mathcal{T}_{\text {no-comm }}$ and probability estimate $p_{u}$ are the same as in the previous section. However, notice that in the Strong-CD model each state in $\mathcal{T}_{\text {no-comm }}$ has exactly four children, corresponding to all valid combinations of $\left\{\lambda_{S}, \lambda_{N}\right\}$ and \{transmit, listen, idle $\}$ : (transmit, $\left.\lambda_{N}\right)$, (listen, $\left.\lambda_{S}\right)$, (listen, $\left.\lambda_{N}\right)$, and (idle, N/A). Notice that a device transmitting a message never hear silence in the Strong-CD model. The definition of the checkpoints $d_{i}$ and how we select the network sizes $n_{i}$ are also the same as in the previous section.

For an index $i$, the subtree $\mathcal{T}_{i}$, with the sequence $\left\{m_{i, t}\right\}_{1 \leq t \leq d_{i}-1}$ indicating noise/silence of the channel at time slot $t$, is defined inductively as follows. Initially $\mathcal{T}_{i}$ consists of the initial state (the base case of $t=1$ ). For each $1<t \leq d_{i}$, suppose that $\mathcal{T}_{i}$ has been defined up to layer $t-1$. If there exists a state $v$ in layer $t-1$ of $\mathcal{T}_{i}$ with $p_{v} \cdot p_{v} \leadsto$ transmit $\geq n_{i}{ }^{-1 / 10}$, define $m_{i, t-1}=\lambda_{N}$; otherwise define $m_{i, t-1}=\lambda_{S}$. A state $u$ in layer $t$ that is a child of a state in $\mathcal{T}_{i}$ is added to the subtree $\mathcal{T}_{i}$ if the following two conditions are met:

- The probability estimate of $u$ is high: $p_{u} \geq n_{i}{ }^{-1 / 10}$.

- Either the action performed in the first phase of time slot $t-1$ that leads to the state $u$ is idle, or the message received in the second phase of time slot $t-1$ that leads to the state $u$ is $m_{i, t-1}$.

Observe that all states in $\mathcal{T}_{i}$ are confined to layer at most $d_{i}$, and each state in $\mathcal{T}_{i}$ that is not a leaf has 3 children, corresponding to the three actions.

Similar to the previous section, for an execution of $\mathcal{A}$ on $n_{i}$ devices, we write $\mathcal{P}_{i}$ to denote the event that for each state $u$ in layer $1 \leq t \leq d_{i}$ of the decision tree $\mathcal{T}$, the number of devices entering $u$ is within $n_{i} \cdot p_{u} \pm t \cdot n_{i}{ }^{0.6}$ if $u$ is in layer $t$ of $\mathcal{T}_{i}$, and is 0 if $u \notin \mathcal{T}_{i}$.

Lemma 3.7. For an execution of $\mathcal{A}$ on $n_{i}$ devices, $\mathcal{P}_{i}$ holds with probability at least $1-n_{i}{ }^{-7}$.

Proof. This is essentially the same as the proof of Lemma 3.5.

High Energy Path and Active Indices. Observe that, unlike the Sender-CD model, we do not have $\mathcal{T}_{1} \subseteq \mathcal{T}_{2} \subseteq \ldots$. Therefore, to obtain a lower bound, a new technique is needed. Let $k \geq 3$ be an integer. Among $\left\{n_{i}\right\}_{1 \leq i \leq k}$, our goal is to find an index $\hat{i}$ such that for an execution of $\mathcal{A}$ on $n_{\hat{i}}$ devices, with probability $1-1 / \operatorname{poly}\left(n_{\hat{i}}\right)$ there exists a device that uses $\Omega(\log k)$ unit of energy. This is achieved by constructing a high energy path $P=\left(u_{1}, u_{2}, \ldots, u_{\hat{t}}\right)$, along with a sequence of sets of active indices $\left\{K_{t}\right\}_{1 \leq t \leq \hat{t}}$ such that $i \in K_{t}$ implies $u_{t} \in \mathcal{T}_{i}$. The path $P$ is a directed path in the tree $\mathcal{T}_{\text {no-comm }}$, and $u_{t}$ belongs to layer $t$, for each $t$. The number $\hat{t}$ will de chosen later. Intuitively, any device entering the state $u_{t}$ is unable to distinguish between $\left\{n_{i}\right\}_{i \in K_{t}}$, and we will later see that $\{1, \ldots, k\}=K_{1} \supseteq K_{2} \supseteq \ldots$

The path $P$ is chosen to contain at least $\Omega(\log k)$ transitions that corresponds to listen or transmit. Therefore, by setting $\hat{i}$ as any index in $K_{\hat{t}}$, we have $u_{\hat{t}} \in \mathcal{T}_{\hat{i}}$, and hence $\hat{t} \leq d_{\hat{i}}$. By Lemma 3.7, in an execution of $\mathcal{A}$ on $n_{\hat{i}}$ devices, with probability $1-n_{\hat{i}}^{-7}$ there is at least $n_{\hat{i}} \cdot p_{u_{\hat{t}}}-\hat{t} \cdot n_{\hat{i}}{ }^{0.6}=\Omega\left(n_{\hat{i}}{ }^{0.9}\right)>1$ device entering the state $u_{\hat{t}}$, and such a device uses at least $\Omega(\log k)$ unit of energy.

One may attempt to construct the path $P$ by a greedy algorithm which iteratively extends the path by choosing the child state with the highest probability estimate. But this is insufficient to warrant any energy expenditure in $P$. To force an energy expenditure in $P$, we make use of the property that a state entering $u_{t}$ is unable to distinguish between the network sizes in $\left\{n_{i}\right\}_{i \in K_{t}}$ (recall that $i \in K_{t}$ implies $u_{t} \in \mathcal{T}_{i}$ ). If $i \in K_{d_{i}}$, the probability that a device $s$ entering the state $u_{d_{i}}$ remains idle in all the time slots $\left\{d_{i}, \ldots, d_{i+1}-1\right\}$ must be small. Notice that for an execution of $\mathcal{A}$ to be successful, the device $s$ needs to know whether the underlying network size is $n_{i}$ by the time constraint $T\left(n_{i}\right)<d_{j+1}$. In light of the above, in our construction of $P$, a special update rule, which ensures one energy expenditure in the time interval $\left[d_{i}, d_{i+1}-1\right]$, is invoked when a checkpoint $d_{i}$ is met with $i \in K_{d_{i}}$. 
Formally, the high energy path $P$ and the sequence of sets of active indices $\left\{K_{t}\right\}$ are defined by the following procedure: Initially $P=\left(u_{1}\right)$ contains only the initial state, and $K_{1}=\{1,2, \ldots, k\}$. The following update rules are applied repeatedly until either a terminal state is reached or $K_{t}=\emptyset$.

Let the current $P$ be $\left(u_{1}, u_{2}, \ldots, u_{t}\right)$.

- Case "regular update": $t \neq d_{i}$ for all $i \in K_{t}$. Let $x \in$ \{transmit, listen, idle\} be chosen to maximize $p_{u_{t} \rightsquigarrow x}$. If $x=$ idle, append the child of $u_{t}$ that corresponds to being idle at time slot $t$ to the end of $P$, and set $K_{t+1}=K_{t}$. Otherwise, let $m \in\left\{\lambda_{S}, \lambda_{N}\right\}$ be chosen to maximize the number of indices $j \in K_{t}$ with $m_{j, t}=m$, and append the child of $u_{t}$ that corresponds to performing action $x$ and receiving message $m$ at time slot $t$ to the end of $P$, and set $K_{t+1}$ to the set of indices $j \in K_{t}$ with $m_{j, t}=m$.

- Case "special update": $t=d_{i}$ for some $i \in K_{t}$. Let $t^{\prime} \in$ $\left\{d_{i}+1, \ldots, d_{i+1}\right\}$ and $x \in\{$ transmit, listen $\}$ be chosen to maximize the probability for a device entering $u_{t}$ to remain idle during all the time slots $\left\{t, \ldots, t^{\prime}-2\right\}$ and to perform $x$ in time slot $t^{\prime}-1$. Let $m \in\left\{\lambda_{S}, \lambda_{N}\right\}$ be chosen to maximize the number of indices $j \in K_{t} \backslash\{i\}$ with $m_{j, t^{\prime}-1}=m$. We let $u_{t^{\prime}}$ be the unique descendant of $u_{t}$ resulting from applying $t^{\prime}-t$ idle actions during time slots $t, \ldots, t^{\prime}-2$, and then performing action $x$ and receiving message $m$ at time slot $t^{\prime}-1$. The path $P$ is extended to have $u_{t^{\prime}}$ as the new end point. For each $t^{\prime \prime} \in\left\{t+1, \ldots, t^{\prime}\right\}$, we let $K_{t^{\prime \prime}}$ to be the set of indices $j \in K_{t} \backslash\{i\}$ with $m_{j, t^{\prime}-1}=m$.

We select $\hat{t}$ to be the largest number such that $K_{\hat{t}} \neq \emptyset$. See Figure 4 for an illustration of the update rules. Notice that, in the special update, the reason $i$ must be removed from the set of the active indices is that $\mathcal{T}_{i}$ only contains states up to layer $d_{i}$.

The Lower Bound. To obtain the desired lower bound, we need to show that (i) $i \in K_{t}$ implies $u_{t} \in \mathcal{T}_{i}$, and that (ii) the energy cost of the path $P$ is $\Omega(\log k)$. To prove (i), we need to lower bound the probability estimate of the states in $P$. We observe the following:

- In a regular update at time $t$, we have $p_{u_{t+1}} \geq \frac{1}{3} p_{u_{t}}$.

- In a special update at time $t=d_{j}$, we have:

$$
p_{u_{t^{\prime}}} \geq \frac{p_{u_{t}}}{2 \cdot\left(d_{j+1}-d_{j}\right)}\left(1-p_{\text {idle }}\right)>\frac{p_{u_{t}}}{2 d_{j+1}}\left(1-p_{\text {idle }}\right),
$$

where $p_{\text {idle }}$ is defined as the probability that a device entering the state $u_{d_{j}}$ is idle in all the time slots $\left\{d_{j}, \ldots, d_{j+1}-\right.$ $1\}$.

Lemma 3.8. For each $i \in\{1, \ldots, k\}$ and each $t \in\{1, \ldots, \hat{t}\}$ such that $i \in K_{t}$, we have $u_{t} \in \mathcal{T}_{i}$.

Proof. Suppose that by induction hypothesis the statement of the lemma holds for all $i^{\prime} \leq i$ and $t^{\prime} \leq t$ such that $(i, t) \neq$ $\left(i^{\prime}, t^{\prime}\right)$. Proving $p_{u_{t}} \geq\left(2^{2^{d_{i}}}\right)^{-1 / 10}>n_{i}{ }^{-1 / 10}$ suffices to guarantee that $u_{t} \in \mathcal{T}_{i}$. Notice that the procedure constructing $P$ and $\left\{K_{t}\right\}$ guarantees that $i \in K_{t}$ implies that either (i) $u_{t-1}$ is idle, or (ii) the message received at time slot $t-1$ that leads to the state $u_{t}$ is $m_{i, t-1}$. Moreover, the special update implies that $t \leq d_{i}$.
We claim that $p_{\text {idle }}<\frac{2}{n_{j}}<1 / 2$. This implies that $p_{u_{t^{\prime}}} \geq \frac{p_{u_{t}}}{4 d_{j+1}}$ in the special update at time $t=d_{j}$. Therefore, by the above two observations, $p_{u_{t}}$ is at least

$$
3^{-t} \prod_{j=1}^{\arg \max _{j^{\prime}}\left\{d_{j^{\prime}}<t\right\}} \frac{1}{4 \cdot d_{j+1}}=3^{-d_{i}} \cdot\left(\frac{1}{4 \cdot d_{i}}\right)^{i-1}>\left(2^{2^{d_{i}}}\right)^{-1 / 10} .
$$

Hence the lemma holds (notice that $\arg \max _{j^{\prime}}\left\{d_{j^{\prime}}<t\right\}=O\left(\log ^{*} t\right)$, and also $t \leq d_{i}$ ).

What remains to do is to prove that $p_{\text {idle }}<2 / n_{j}$ when a special update is applied at time $d_{j}$. Let $v$ be the state at time $d_{j+1}$ that results from being idle in time slots $\left\{d_{j}, \ldots, d_{j+1}-1\right\}$ after leaving the state $u_{d_{j}}$. Since a special update is applied at time $d_{j}$, we have $j \in K_{d_{j}}$. Also, the fact that $i \in K_{t}$ guarantees $i \in K_{d_{j}}$. Therefore, by induction hypothesis, $u_{d_{j}}$ belongs to both $\mathcal{T}_{i}$ and $\mathcal{T}_{j}$. Since $d_{j}<t \leq$ $d_{i}, i$ and $j$ are distinct indices.

- If $v$ does not give a correct estimate of $n_{j}$, then we have $1 / n_{j} \geq\left(1-n_{j}{ }^{-7}\right) \cdot p_{\text {idle }}$, since otherwise Lemma 3.7 implies that with probability higher than $1 / n_{j}$ there is a device in the state $v$ when we execute $\mathcal{A}$ on $n_{j}$ devices, and such a device does not give a correct estimate of $n_{j}$ by time $T\left(n_{j}\right)<d_{j+1}$.

- Otherwise, the devices entering the state $v$ have made a decision, and such a decision is not a correct estimate of $n_{i}$. Similarly we need to have $1 / n_{i} \geq\left(1-n_{i}{ }^{-7}\right) \cdot p_{\text {idle }}$.

As a result, $p_{\text {idle }} \leq \frac{1}{n_{j} \cdot\left(1-n_{j}^{-7}\right)}<\frac{2}{n_{j}}$.

To lower bound the energy cost of $P$, we observe the following:

- In a regular update at time $t$, either (i) $\left|K_{t+1}\right|=\left|K_{t}\right|$, or (ii) $\left|K_{t+1}\right| \geq\left|K_{t}\right| / 2$, and there is one unit of energy expenditure at time slot $t$.

- In a special update at time $t=d_{j}$, for each $t^{\prime \prime} \in\{t+$ $\left.1, \ldots, t^{\prime}\right\},\left|K_{t^{\prime \prime}}\right| \geq\left(\left|K_{t}\right|-1\right) / 2$, and there is one unit of energy expenditure at time slot $t^{\prime}-1$.

Therefore, the total energy expenditure in the path $P=\left(u_{1}, \ldots, u_{\hat{t}}\right)$ is $\Omega\left(\log \left|K_{1}\right|-\log \left|K_{\hat{t}}\right|\right)$. As long as $\left|K_{\hat{t}}\right| \leq 2$, the energy cost is at least $\Omega\left(\log k\right.$ ) (recall that $\left|K_{1}\right|=k$ ). The only possibility to cause $\left|K_{\hat{t}}\right|>2$ is that $u_{\hat{t}}$ is a terminal state, and so no more update rule can be applied to extend the path $P$ any further. However, by Lemma 3.8, $u_{\hat{t}} \in \mathcal{T}_{i}$ for each $i \in K_{\hat{t}}$, and hence Lemma 3.7 guarantees that, with probability $1-n_{i}{ }^{-7}$, there is a device $s$ that enters $u_{\hat{t}}$ when $\mathcal{A}$ is executed on $n_{i}$ devices. If $u_{\hat{t}}$ is a terminal state, then there must exist some $i \in K_{\hat{t}}$ such that $s$ gives an incorrect estimate of $n_{i}$. Therefore, $\left|K_{\hat{t}}\right| \leq 2$, and we conclude the theorem.

TheORem 3.9. Let $k \geq 3$ be any integer. Then there exists a network size $n$ with $d_{1} \leq n \leq d_{k+1}$ such that if $\mathcal{A}$ is executed on $n$ devices, with probability at least $1-n^{-7}$ there exists a device $s$ that uses $\Omega(\log k)$ units of energy.

As long as $T(n)=O(\overbrace{2^{2^{2^{n}}}}^{\alpha})$ for a constant $\alpha$ and $T(n) \geq n$, the energy cost of Approximate Counting in the Sender-CD model is $\Omega\left(\log \left(\log ^{*} n\right)\right)$. Similarly, the lower bound generalizes to higher time constraints as follows: For any function $g(n) \leq \log n$, under time constraint $T(n)=g^{-1}(n)$, the energy cost is $\Omega\left(\log \left(g^{*}(n)\right)\right)$. 

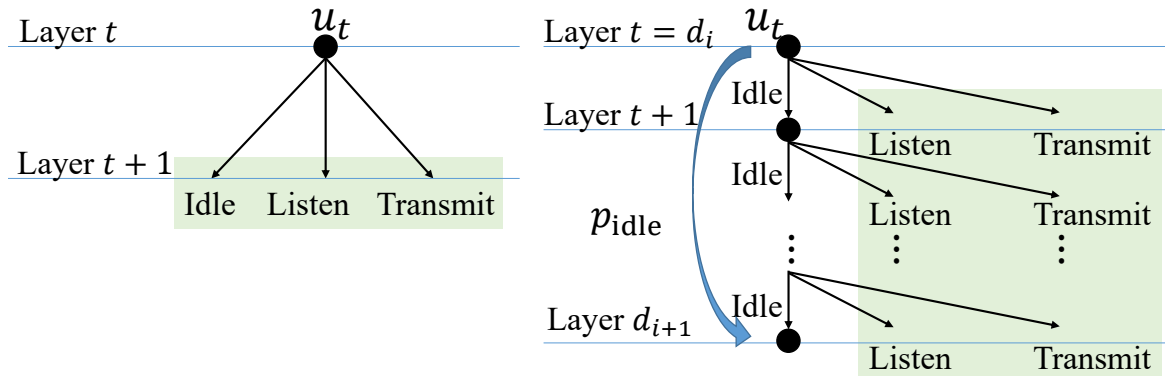

Figure 4: Left: regular update. Right: special update. The shaded region indicates the set of candidate endpoints to extend the current path $P$.

\subsection{Lower Bound for Other Problems}

In this section we demonstrate how our lower bounds proofs can be adapted to any problem that requires a device at least successfully transmit or listen once before it terminates. This implies that our lower bounds apply to Leader Election and the contention resolution problem studied in [7].

Consider the Sender-CD model. Let $\mathcal{A}$ be an algorithm which takes $T(n)$ time, and a device in an execution of $\mathcal{A}$ must successfully transmit or listen at least once before it terminates. Let $n=n_{i}$ for some $i$. By Lemma 3.5, with probability at least $1-n_{i}{ }^{-7}$, the runtime of all devices in an execution of $\mathcal{A}$ is at least $d_{i}$.

The runtime of a device in an execution of $\mathcal{A}$ (which is at least $d_{i}$ and at most $\left.T\left(n_{i}\right)<d_{i+1}\right)$ can be seen as a very loose estimate of the network size $n=n_{i}$. Hence $\mathcal{A}$ actually solves a relaxed version of Approximate Counting (for $n=n_{i}$ ) such that (i) any estimate $d_{i} \leq \tilde{n}<d_{i+1}$ suffices, and (ii) different devices are allowed to have different estimates.

Since one estimate $\tilde{n}$ only works for at most one $n_{i}$, the lower bound proof for Theorem 3.1 still applies in such a relaxed setting. ${ }^{4}$ Similarly, for the Strong-CD model, Theorem 3.2 applies to any problem that requires a device at least successfully transmit or listen once before it terminates.

\section{DETERMINISTIC ALGORITHMS FOR LEADER ELECTION AND CENSUS}

In this section we prove a tight upper bound on the deterministic energy complexity of Census in Sender-CD, which can also be applied to solve Leader Election. Our algorithms are inspired by the energy-sharing technique of Jurdzinski et al. [22].

Theorem 4.1. There exists a deterministic Sender-CD algorithm that solves Leader Election and Census in $O(N)$ time with energy $O(\log \log N)$.

\subsection{Groups in Deterministic Algorithms}

We first introduce the concept of groups, which will be used by our deterministic algorithms. A group is an ordered list $\left(s_{0}, s_{1}, \ldots, s_{k-1}\right)$ of active device IDs and each device is in at most one group. The group containing a device $s_{i}$ is denoted $G=G\left(s_{i}\right)$ and its rank is

\footnotetext{
${ }^{4}$ Also observe that our lower bounds work for estimating $\log { }^{*} n$ within a constant additive error
}

$r\left(s_{i}\right)=i$. The size of the group is $|G|=k$. The representative device $s_{0}$ is called the master of $G$, denoted $m(G)$. Each group has a unique group ID, and each device $s_{i}$ knows the group ID of $G\left(s_{i}\right), m\left(G\left(s_{i}\right)\right)$, $\left|G\left(s_{i}\right)\right|$ and $r\left(s_{i}\right)$.

The notion of groups already breaks symmetry: if an algorithm outputs a single group, the current master of this group can be considered the leader. To solve the more difficult Census problem, we formalize the information known by a device $s$ as a set $I(s)$ maintained by $s$.

Definition 4.2. A group $G$ is centralized if $I(m(G))=\bigcup_{s \in G} I(s)$.

Notice that, by initializing $I(s)=\{s\}$ for each active device ID $s$, the Census problem is solved when there is one device collecting $\{I(s)\}_{s}$ over all active IDs $s$.

\subsection{A Simple Census/Leader Election Algorithm}

In this section we show how to leverage the notion of groups in order to distribute energy costs, by presenting a simple deterministic Census algorithm SimpleCensus $(\hat{N})$. Prior to executing the algorithm, it is guaranteed that the devices are partitioned into centralized groups with group ID space $[\hat{N}]$, and each group has size larger than $\log \hat{N}$. After the execution of SimpleCensus $(\hat{N})$, one device $s$ of rank $h=\lceil\log \hat{N}\rceil$ identifies itself as the leader which collects all input information.

The algorithm SimpleCensus $(\hat{N})$ is executed recursively. If $\hat{N}=$ 1 , the leader device is simply the master of the only group. If $\hat{N}>1$, the algorithm recursively calls SimpleCensus $(\lceil\hat{N} / 2\rceil)$ two times on the two halves of the ID space, respectively. Suppose the two leader devices of the two recursive calls are $s_{0}$ and $s_{1}$, which both have rank $h-1$ in their own groups.

In the final two time slots, $s_{0}$ announces its group ID and $I\left(s_{0}\right)$, and $s_{1}$ does likewise. Each device with rank $h$ in its group listens to these two slots. If $s_{0}$ exists, the leader is the device in $G\left(s_{0}\right)$ of rank $h$; if $s_{0}$ does not exist and $s_{1}$ exists, the leader is the device in $G\left(s_{1}\right)$ with rank $h$. The leader device $s$ then sets $I(s) \leftarrow I\left(s_{0}\right) \cup I\left(s_{1}\right)$. Observe that any device listens to at most two time slots and transmits in at most one.

THeOREM 4.3. The algorithm SimpleCensus $(\hat{N})$ successfully assigns a leader device s in $O(\hat{N})$ time, where $I(s)$ contains $\bigcup_{G} I(m(G))$ for every group $G$. Moreover, in each group $G$, devices with ranks 


\section{$0,1, \ldots,\lceil\log \hat{N}\rceil$ spend constant energy and all others spend zero energy.}

The algorithm SimpleCensus $(N)$ solves Census and Leader Election efficiently, and the strategy behind our proofs of Theorem 4.1 is to merge the devices into groups to $\lceil\log N\rceil$, so that an application of SimpleCensus $(N)$ solves the problem.

\subsection{An $O(\log \log N)$ Census/Leader Election Algorithm}

In this section we prove Theorem 4.1. We start with an algorithm that solves Leader Election using $O(\log \log N)$ energy and $O(N)$ time. At the end of this section, we generalize this algorithm to solve Census, still using $O(\log \log N)$ energy and $O(N)$ time.

Without loss of generality, we assume $\log N$ is an integer. Our algorithm consists of $\log \log N$ phases. All devices participate initially and may drop out in the middle of the algorithm. We maintain the following invariants:

(1) At the beginning of the $i$-th phase, all participating devices are organized into active groups of size exactly $2^{i}$.

(2) The number of active groups is at least one.

At the beginning of Phase 0 , each device forms a singleton group. During each phase, some pairs of groups are merged into groups of doubled size; the devices in the unmerged groups are terminated. There are two possible outcomes of our algorithm.

- After Phase $i$ there is only one active group $G$ remaining, for some $i \leq \log \log N-1$. Then the algorithm is terminated at Phase $i$ with the master of $G$ being the leader.

- More than one active group remains after Phase $\log \log N-$ 1. As the groups that survive until the end have size $\log N$, a leader can be elected by applying SimpleCensus $(N)$.

Intuitively, in Phase $i$ of our algorithm, each active group attempts to find another active group to merge into a group with size $2^{i+1}$. All groups that are not merged during Phase $i$ are terminated. We will later see that the energy cost per active group is $O(\log \log N)$. For each phase, there is a representative in each group which is responsible for carrying out the procedure in this phase. At the end of a phase the representative of a group announces their new group membership to other group members. The energy cost in a phase for a device $s$ is $O(\log \log N)$ if $s$ serves as a representative in this phase, and is $O(1)$ otherwise. We will later see that each device is responsible for no more than 2 phases throughout the algorithm. Therefore, the total energy cost of the algorithm is

$$
2 \cdot O(\log \log N)+\sum_{i=0}^{\log \log N-1} O(1)=O(\log \log N) .
$$

In the subsequent discussion we focus on describing and analyzing one phase of the algorithm, which is based on the procedure $\operatorname{Det} L E(\hat{N})$. Prior to executing the procedure $\operatorname{Det} L E(\hat{N})$, it is guaranteed that the devices are partitioned into centralized groups with group ID space $[\hat{N}]$, each group has the same size, and the number of groups is at least 2. By the end of the execution, each group has either dropped out or merged with another group. The procedure $\operatorname{Det} L E(\hat{N})$ is defined recursively as follows.

Base Case. There are exactly two groups $G_{0}$ and $G_{1}$, and $\hat{N}=2$. Using two time slots, the representatives of the two groups exchange the information $I\left(G_{0}\right)$ and $I\left(G_{1}\right)$. Then the two groups are merged.

Inductive Step. Uniformly divide the group ID space $[\hat{N}]$ into $\hat{N}^{\prime}=\lceil\sqrt{\hat{N}}\rceil$ intervals. For each $j \in\left[\hat{N}^{\prime}\right]$, if there are at least two groups whose ID belongs to the $j$-th interval of $\hat{N}^{\prime}$ IDs (this can be checked in one time slot), recursively call $\operatorname{Det} L E\left(\hat{N}^{\prime}\right)$ on the $j$-th interval of $\hat{N}^{\prime}$ IDs. Each group that does not participate in any recursive call change its ID from $d$ to $\left\lfloor d / \hat{N}^{\prime}\right\rfloor$. If the number of remaining groups is at least two (again, this can be checked in one time slot), then call $\operatorname{DetLE}\left(\hat{N}^{\prime}\right)$ on these groups.

By initially setting $\min _{s \in G} \operatorname{ID}(s)$ as the group ID of each active group $G$, an execution of $\operatorname{Det} L E(N)$ fulfills the task of one phase of our algorithm. The inductive step guarantees that (i) each recursive call invoked has at least two groups participating, and (ii) as long as the number of active groups is at least 2 , at least one recursive call is invoked. Therefore, given that the number of active groups is at least 2 in the beginning of a phase, we must reach the base case and have two groups merged.

Let $E(N)(T(N))$ denote the energy complexity (time complexity) of $\operatorname{Det} L E(N)$. Since each representative is involved in at most one recursive call of $\operatorname{DetLE}(\lceil\sqrt{\hat{N}}\rceil)$, we have $E(\hat{N})=E(\lceil\sqrt{\hat{N}}\rceil)+O(1)$. Hence the energy cost is $O(\log \log N)$. Similarly, since $\operatorname{DetLE}(N)$ invokes at most $\lceil\sqrt{\hat{N}}\rceil+1$ recursive calls of $\operatorname{DetLE}(\lceil\sqrt{\hat{N}}\rceil)$, we have $T(\hat{N})=(\lceil\sqrt{\hat{N}}\rceil+1) \cdot T(\lceil\sqrt{\hat{N}}\rceil)+O(\lceil\sqrt{\hat{N}}\rceil)$. Hence the runtime is $O(N)$.

Energy Sharing. As mentioned earlier, in order to save energy, only the representatives of the groups participate in the procedure of each phase. For Phase $i(i>0)$, the device with rank $2^{i-1}-1$ in group $G$ will be selected as the representative of $G$. It is straightforward to verify that, after Phase 0 , each device $s$ serves as a representative for at most one time.

We allocate $N$ extra time slots after each phase to let the representatives announce their new group membership to other group members. Let $G$ and $G^{\prime}$ be two groups that are merged in phase $i$, and let $s\left(s^{\prime}\right)$ be the representative of the group $G\left(G^{\prime}\right)$ in phase $i$. If the ID of the group $G\left(G^{\prime}\right)$ is $g\left(g^{\prime}\right)$, then $s\left(s^{\prime}\right)$ announces the ID of the merged group $G \cup G^{\prime}$ to all other members in $G\left(G^{\prime}\right)$ at the $g$-th $\left(g^{\prime}\right.$-th) time slot. Each device in the merged group then recomputes their new ranks and updates the group size locally. The message size complexity is $O(\log N)$. Also, if $G$ is not successfully merged during phase $i$, the representative of $G$ can also transmit to terminate all devices in $G$.

Time Complexity. Recall that there are $O(\log \log N)$ phases during the execution of the algorithm, each uses $O(N)$ time slots, thus the total time complexity of the algorithm is $O(N \log \log N)$. The running time can be further reduced to $O(N)$ by a preprocessing which uniformly divides the ID space into $\frac{N}{\log \log N}$ intervals and calls SimpleCensus $(\log \log N)$ on every interval. Here the initial group size is 1 , and each device simulates $\log (\log \log N)$ devices in its group. The preprocessing uses $O(N)$ time and $O(\log \log \log N)=$ $o(\log \log N)$ energy, and after that the leader devices from the 
$\frac{N}{\log \log N}$ intervals execute the algorithm in this section on the ID space $\left[\frac{N}{\log \log N}\right]$.

Solving Census. Now we generalize DetLE to solve Census. In order to solve Census, we only need to record the information of those groups that are dropped out during each phase. Recall that during the execution of DetLE, a group $G$ is dropped out if and only if $G$ does not participate in any recursive call at some inductive step. It is clear from the description of DetLE that $G$ must be the only group that does not participate in any recursive call at that inductive step. Furthermore, DetLE guarantees that after that inductive step, at least one of pair of groups are merged. Thus, we add one time slot at the end of each inductive step of DetLE, in which the only group that did not participate in any recursive call transmits its group information while all merged groups listen. The information of dropped out groups can hence be recorded and merged during further steps.

\section{ACKNOWLEDGMENTS}

We would like to thank Tomasz Jurdziński for discussing the details of [22-25] and to Calvin Newport for assisting us with the radio network literature.

\section{REFERENCES}

[1] N. Alon, A. Bar-Noy, N. Linial, and D. Peleg. 1991. A lower bound for radio broadcast. F. Comput. System Sci. 43, 2 (1991), 290-298.

[2] R. Bar-Yehuda, O. Goldreich, and A. Itai. 1991. Efficient emulation of single-hop radio network with collision detection on multi-hop radio network with no collision detection. Distributed Computing 5, 2 (1991), 67-71.

[3] R. Bar-Yehuda, O. Goldreich, and A. Itai. 1992. On the time-complexity of broadcast in multi-hop radio networks: An exponential gap between determinism and randomization. F. Comput. System Sci. 45, 1 (1992), 104-126.

[4] M. Barnes, C. Conway, J. Mathews, and D. K. Arvind. 2010. ENS: An Energy Harvesting Wireless Sensor Network Platform. In Proceedings of the 5th International Conference on Systems and Networks Communications. 83-87. DOI http://dx.doi.org/10.1109/ICSNC.2010.18

[5] M. A. Bender, J. T. Fineman, S. Gilbert, and M. Young. 2016. How to Scale Exponential Backoff: Constant Throughput, Polylog Access Attempts, and Robustness. In Proceedings of the 27th Annual ACM-SIAM Symposium on Discrete Algorithms (SODA). 636-654. DOI : http://dx.doi.org/10.1137/1.9781611974331.ch47

[6] M. A. Bender, J. T. Fineman, M. Movahedi, J. Saia, V. Dani, S. Gilbert, S. Pettie, and M. Young. 2015. Resource-Competitive Algorithms. SIGACT News 46, 3 (2015), 57-71. DOI : http://dx.doi.org/10.1145/2818936.2818949

[7] M. A. Bender, T. Kopelowitz, S. Pettie, and M. Young. 2016. Contention Resolution with Log-logstar Channel Accesses. In Proceedings of the 48th Annual ACM Symposium on Theory of Computing (STOC). 499-508. DOI : http://dx.doi.org/10 $1145 / 2897518.2897655$

[8] P. Brandes, M. Kardas, M. Klonowski, D. Pajak, and R. Wattenhofer. 2016. Approximating the Size of a Radio Network in Beeping Model. In Proceedings of the 23rd International Colloquium on Structural Information and Communication Complexity (SIROCCO).

[9] Yi-Jun Chang, Tsvi Kopelowitz, Seth Pettie, Ruosong Wang, and Wei Zhan. 2016. Exponential Separations in the Energy Complexity of Leader Election. CoRR abs/1609.08486 (2016). http://arxiv.org/abs/1609.08486

[10] B. S. Chlebus, D. R. Kowalski, and A. Pelc. 2012. Electing a leader in multi-hop radio networks. In The 16th International Conference On Principles Of Distributed Systems (OPODIS). Springer, 106-120.

[11] A. E. F. Clementi, A. Monti, and R. Silvestri. 2003. Distributed broadcast in radio networks of unknown topology. Theoretical Computer Science 302, 1 (2003), 337-364.

[12] A. Cornejo and F. Kuhn. 2010. Deploying wireless networks with beeps. In Proceedings of The 24th International Symposium on Distributed Computing (DISC) Springer, 148-162.

[13] A. Czumaj and P. Davies. 2016. Brief Announcement: Optimal Leader Election in Multi-Hop Radio Networks. In Proceedings 35th ACM Symposium on Principles of Distributed Computing (PODC). 47-49. DOI : http://dx.doi.org/10.1145/2933057. 2933076
[14] A. Czumaj and W. Rytter. 2003. Broadcasting algorithms in radio networks with unknown topology. In Proceedings of the 44th Annual IEEE Symposium on Foundations of Computer Science (FOCS). 492-501.

[15] S. Daum, S. Gilbert, F. Kuhn, and C. Newport. 2012. Leader election in shared spectrum radio networks. In Proceedings of the 31st ACM symposium on Principles of distributed computing (PODC). 215-224.

[16] P. Erdős, A. Rényi, and V. T. Sós. 1966. On a problem of graph theory. Studia Sci. Math. Hung. 1 (1966), 215-235.

[17] M. Farach-Colton, R. J. Fernandes, and M. A. Mosteiro. 2006. Lower Bounds for Clear Transmissions in Radio Networks. In Proceedings of the 7th Latin American Symposium on Theoretical Informatics (LATIN). 447-454. DOI: http: //dx.doi.org/10.1007/11682462 42

[18] M. Ghaffari and B. Haeupler. 2013. Near optimal leader election in multi-hop radio networks. In Proceedings of the 24th Annual ACM-SIAM Symposium on Discrete Algorithms (PODC). 748-766.

[19] S. Gilbert, V. King, S. Pettie, E. Porat, J. Saia, and M. Young. 2014. (Near) optimal resource-competitive broadcast with jamming. In Proceedings of the 26th ACM Symposium on Parallelism in Algorithms and Architectures (SPAA). 257-266. DOI : http://dx.doi.org/10.1145/2612669.2612679

[20] S. Gilbert and C. Newport. 2015. The computational power of beeps. In Proceedings of the 29th International Symposium on Distributed Computing (DISC). Springer, 31-46.

[21] A. G Greenberg and S. Winograd. 1985. A lower bound on the time needed in the worst case to resolve conflicts deterministically in multiple access channels. 7. ACM 32, 3 (1985), 589-596.

[22] T. Jurdzinski, M. Kutylowski, and J. Zatopianski. 2002. Efficient algorithms for leader election in radio networks. In Proceedings of the 21st Annual ACM Symposium on Principles of Distributed Computing (PODC). 51-57. DOI : http: //dx.doi.org/10.1145/571825.571833

[23] T. Jurdzinski, M. Kutylowski, and J. Zatopianski. 2002. Energy-Efficient Size Approximation of Radio Networks with No Collision Detection. In Proceedings of the 8th Annual International Conference on Computing and Combinatorics (COCOON). 279-289. DOI : http://dx.doi.org/10.1007/3-540-45655-4_31

[24] T. Jurdzinski, M. Kutylowski, and J. Zatopianski. 2002. Weak Communication in Radio Networks. In Proceedings of the 8th International European Conference on Parallel Computing (Euro-Par). 965-972. DOI : http://dx.doi.org/10.1007/ 3-540-45706-2_137

[25] T. Jurdzinski, M. Kutylowski, and J. Zatopianski. 2003. Weak communication in single-hop radio networks: adjusting algorithms to industrial standards. Concurrency and Computation: Practice and Experience 15, 11-12 (2003), 1117-1131. DOI : http://dx.doi.org/10.1002/cpe.783

[26] T. Jurdzinski and G. Stachowiak. 2002. Probabilistic Algorithms for the Wakeup Problem in Single-Hop Radio Networks. In Proceedings of the 13th International Symposium on Algorithms and Computation (ISAAC). 535-549. DOI : http://dx.doi. org/10.1007/3-540-36136-7_47

[27] M. Kardas, M. Klonowski, and D. Pajak. 2013. Energy-efficient leader election protocols for single-hop radio networks. In Proceedings of the 42nd International Conference on Parallel Processing. 399-408.

[28] G. Katona and E. Szemerédi. 1967. On a problem of graph theory. Studia Scientiarum Mathematicarum Hungarica 2 (1967), 23-28.

[29] V. King, J. Saia, and M. Young. 2011. Conflict on a communication channel. In Proceedings of the 30th Annual ACM Symposium on Principles of Distributed Computing (PODC). 277-286. DOI : http://dx.doi.org/10.1145/1993806.1993855

[30] D. R. Kowalski and A. Pelc. 2005. Broadcasting in undirected ad hoc radio networks. Distributed Computing 18, 1 (2005), 43-57. DOI : http://dx.doi.org/10. 1007/s00446-005-0126-7

[31] D. R. Kowalski and A. Pelc. 2009. Leader Election in Ad Hoc Radio Networks: A Keen Ear Helps. In Proceedings of the 36th International Colloquium on Automata, Languages and Programming (ICALP). 521-533. DOI : http://dx.doi.org/10.1007/ 978-3-642-02930-1_43

[32] E. Kushilevitz and Y. Mansour. 1998. An $\Omega(D \log (N / D))$ Lower Bound for Broadcast in Radio Networks. SIAM f. Comput. 27, 3 (1998), 702-712. DOI : http://dx.doi.org/10.1137/S0097539794279109

[33] M. Kutyłowski and W. Rutkowski. 2003. Adversary immune leader election in ad hoc radio networks. In Proceedings of the 11th European Symposium on Algorithms (ESA). Springer, 397-408.

[34] Y. Lee, S. Bang, I. Lee, Y. Kim, G. Kim, M. H. Ghaed, P. Pannuto, P. Dutta, D. Sylvester, and D. Blaauw. 2013. A Modular $1 \mathrm{~mm}^{3}$ Die-Stacked Sensing Platform With Low Power $\mathrm{I}^{2} \mathrm{C}$ Inter-Die Communication and Multi-Modal Energy Harvesting. IEEE fournal of Solid-State Circuits 48, 1 (2013), 229-243.

[35] K. Nakano and S. Olariu. 2000. Energy-Efficient Initialization Protocols for SingleHop Radio Networks with No Collision Detection. IEEE Trans. Parallel Distrib. Syst. 11, 8 (2000), 851-863. DOI : http://dx.doi.org/10.1109/71.877942

[36] K. Nakano and S. Olariu. 2000. Randomized Initialization Protocols for Ad Hoc Networks. IEEE Trans. Parallel Distrib. Syst. 11, 7 (2000), 749-759. DOI: http://dx.doi.org/10.1109/71.877833 
[37] C. C. Newport. 2014. Radio Network Lower Bounds Made Easy. In Proceedings of the 28th International Symposium on Distributed Computing (DISC). 258-272. DOI : http://dx.doi.org/10.1007/978-3-662-45174-8_18

[38] J. Polastre, R. Szewczyk, and D. Culler. 2005. Telos: enabling ultra-low power wireless research. In Proceedings of the 4th International Symposium on Information Processing in Sensor Networks (IPSN). 364-369. DOI : http://dx.doi.org/10 1109/IPSN.2005.1440950

[39] J. Schneider and R. Wattenhofer. 2010. What Is the Use of Collision Detection (in Wireless Networks)?. In Proceedings 24th International Symposium on Distributed

Computing (DISC). 133-147. DOI : http://dx.doi.org/10.1007/978-3-642-15763-9 14

[40] K. M. Sivalingam, M. B. Srivastava, and P. Agrawal. 1997. Low power link and access protocols for wireless multimedia networks. In Proceedings of the 47th IEEE Conference on Vehicular Technology, Vol. 3. 1331-1335. DOI: http: //dx.doi.org/10.1109/VETEC.1997.605397

[41] D. E. Willard. 1986. Log-Logarithmic Selection Resolution Protocols in a Multiple Access Channel. SIAM 7. Comput. 15, 2 (1986), 468-477. DOI : http://dx.doi.org/ $10.1137 / 0215032$ 